\title{
Verzeichnis der abgekürzt zitierten Literatur
}

\author{
$A / D / S^{6}$ \\ Adler/Düring/Schmaltz, Rechnungslegung und Prüfung der Unter- \\ nehmen (Losebl.) \\ $A / F / R$ \\ $B / B$ \\ $\mathrm{BeBiKo} \mathrm{O}^{8}$ \\ Beermann/Gosch \\ $B / H \mathrm{GmbHG} \mathrm{H}^{19}$ \\ B/H HGB H $^{35}$ \\ Binz/Sorg ${ }^{11}$ \\ Blümich \\ Bordewin/Brandt \\ $B / R / O^{5}$ \\ BStGB \\ Crezelius $L B^{2}$ \\ $D / J / P / W$ \\ D/W \\ $E / H$ \\ Ernst \& Young \\ E/S KStG ${ }^{3}$ \\ $F / P / G$ \\ $F / M$ \\ Frotscher \\ $F / \mathrm{W} / B / S$ \\ $F / \mathrm{W} / K$ \\ Gl/Gür GewStG ${ }^{7}$ \\ Gl $H G B^{7}$ \\ Gosch $^{2}$ \\ $G / K / G$ \\ Helmke/Bauer \\ $H / H / R$ \\ $H / H / S p$ \\ Ahrend/Förster/Rößler, Steuerrecht der betrieblichen Altersversorgung \\ (Losebl.) \\ Bordewin/Brandt, Kommentar zum Einkommensteuergesetz (Losebl.) \\ Beck'scher Bilanz-Kommentar, 8. Aufl. 2012 \\ Beermann/Gosch, Abgabenordnung, Finanzgerichtsordnung (Losebl.) \\ Baumbach/Hueck, GmbH-Gesetz, Kommentar, 19. Aufl. 2010 \\ Baumbach/Hopt, HGB, Kommentar, 35. Aufl. 2012 \\ Binz/Sorg, GmbH \& Co KG, 11. Aufl. 2010 \\ Blümich, Kommentar zu EStG, KStG, GewStG und Nebengesetzen \\ (Losebl.) \\ Bordewin/Brandt, Einkommensteuergesetz, Kommentar, (Losebl.) \\ Blomeyer/Rolfs/Otto, Betriebsrentengesetz, Gesetz zur Verbesserung \\ der betrieblichen Altersversorgung, 5. Aufl. 2010 \\ Bundessteuergesetzbuch - Ein Reformentwurf zur Erneuerung des \\ Steuerrechts, vorgelegt von Paul Kirchhof, 2011 \\ Crezelius, Steuerrecht II, 2. Aufl. 1994 \\ Dötsch/Jost/Pung/Witt, Die Körperschaftsteuer, Kommentar zum \\ KStG, UmwStG und zu den einkommensteuerrechtlichen Vorschriften \\ der Anteilseignerbesteuerung (Losebl.) \\ Debatin/Wassermeyer, Doppelbesteuerung (Losebl.) \\ Ebling/Heuermann, Die Kindergeldauszahlung durch den Arbeitgeber, \\ 1996 \\ Ernst \& Young, Körperschaftsteuergesetz-Kommentar (Losebl.) - \\ früher $A A$, Arthur Andersen \\ Erle/Sauter, Körperschaftsteuergesetz, Kommentar, 3. Aufl. 2010 \\ Felsmann/Pape/Giere/König, Einkommensbesteuerung der Land- und \\ Forstwirte (Losebl.) \\ Frotscher/Maas, Kommentar zum Körperschaftsteuer- und Umwand- \\ lungsteuergesetz (Losebl.) \\ Frotscher, Kommentar zum Einkommensteuergesetz (Losebl.) \\ Flick/Wassermeyer/Baumhoff/Schönfeld, Kommentar zum Außensteuer- \\ recht (Losebl.) \\ Flick/Wassermeyer/Kempermann, DBA Deutschland - Schweiz, \\ Kommentar (Losebl.) \\ Glanegger/Güroff, Kommentar zum GewStG, 7. Aufl. 2009 \\ Glanegger/Kirnberger/Kusterer/Ruß/Selder/Stuhlfelner, Kommentar \\ zum HGB, 7. Aufl. 2007 \\ Gosch, Körperschaftsteuergesetz: KStG, 2. Aufl. 2009 \\ Gosch/Kroppen/Grotherr, DBA-Kommentar (Losebl.) \\ Familienleistungsausgleich (Losebl.) \\ Herrmann/Heuer/Raupach, Kommentar zum Einkommensteuer- und \\ Körperschaftsteuergesetz (Losebl.) \\ Hübschmann/Hepp/Spitaler, Kommentar zur Abgabenordnung und \\ Finanzgerichtsordnung (Losebl.)
}




\begin{tabular}{|c|c|}
\hline$H / M / W$ & Hartz/Meeßen/Wolf, ABC-Führer Lohnsteuer (Losebl.) \\
\hline $\begin{array}{l}\text { Höfer BetrAVG } \\
\text { ArbR }\end{array}$ & $\begin{array}{l}\text { Höfer, Gesetz zur Verbesserung der betrieblichen Altersversorgung, } \\
\text { Band I Arbeitsrecht (Losebl.) }\end{array}$ \\
\hline$H / V / V$ & $\begin{array}{l}\text { Höfer/Veit/Verhuven, Betriebsrentenrecht (BetrAVG) Band II: Steuer- } \\
\text { recht/Sozialabgaben, HGB/IFRS (Losebl.) }\end{array}$ \\
\hline$H / W$ & Heuermann/Wagner, Lohnsteuer (Losebl.) \\
\hline$J a k o b L B^{4}$ & Jakob, Einkommensteuer, 4. Aufl. 2008 \\
\hline Jauernig $^{14}$ & Jauernig, Bürgerliches Gesetzbuch, Kommentar, 14. Aufl. 2011 \\
\hline$K / F / K$ & $\begin{array}{l}\text { Klein/Flockermann/Kühr, Handbuch des Einkommensteuerrechts } \\
\text { (Losebl.) }\end{array}$ \\
\hline Klein ${ }^{10}$ & Klein, Kommentar zur AO, 10. Aufl. 2009 \\
\hline Knobbe-Keuk ${ }^{9}$ & Knobbe-Keuk, Bilanz- und Unternehmenssteuerrecht, 9. Aufl. 1993 \\
\hline Korn & Korn, Einkommensteuergesetz, Kommentar (Losebl.) \\
\hline Kruse & Kruse, Lehrbuch des Steuerrechts, Band I, Allgemeiner Teil, 1991 \\
\hline$K / S / M$ & $\begin{array}{l}\text { Kirchhof/Söhn/Mellinghoff, Einkommensteuergesetz, Kommentar } \\
\text { (Losebl.) }\end{array}$ \\
\hline$K / \mathrm{W}$ & Küting/Weber, Handbuch der Rechnungslegung (Losebl.) \\
\hline Lademann & Lademann, Einkommensteuergesetz, Kommentar (Losebl.) \\
\hline$L / B / P$ & Littmann/Bitz/Pust, Das Einkommensteuerrecht (Losebl.) \\
\hline Leingärtner & Leingärtner, Besteuerung der Landwirte (Losebl.) \\
\hline Märkle/Hiller ${ }^{10}$ & $\begin{array}{l}\text { Märkle/Hiller, Die Einkommensteuer bei Land- und Forstwirten, } \\
\text { 10. Aufl. } 2010\end{array}$ \\
\hline Meincke $e^{15}$ & $\begin{array}{l}\text { Meincke, Erbschaftsteuer- und Schenkungsteuergesetz, Kommentar, } \\
\text { 15. Aufl. } 2009\end{array}$ \\
\hline Palandt $^{71}$ & Palandt, Bürgerliches Gesetzbuch, Kommentar, 71. Aufl. 2012 \\
\hline Schaumburg $^{3}$ & Schaumburg, Internationales Steuerrecht, 3. Aufl. 2011 \\
\hline Schmidt ${ }^{30}$ & Schmidt, Einkommensteuergesetz, Kommentar, 30. Aufl. 2011 \\
\hline$S / K / K$ & $\begin{array}{l}\text { Strunk/Kaminski/Köhler, Außensteuergesetz/Doppelbesteuerungs- } \\
\text { abkommen, Kommentar (Losebl.) }\end{array}$ \\
\hline Streck ${ }^{7}$ & Streck, Körperschaftsteuergesetz, Kommentar, 7. Aufl. 2008 \\
\hline Tiedtke $L B^{2}$ & Tiedtke, Einkommensteuer- und Bilanzsteuerrecht, 2. Aufl. 1995 \\
\hline Tipke/Lang ${ }^{20}$ & Tipke/Lang, Steuerrecht, 20. Aufl. 2010 \\
\hline$T / K$ & $\begin{array}{l}\text { Tipke/Kruse, Abgabenordnung/Finanzgerichtsordnung, Kommentar } \\
\text { (Losebl.) }\end{array}$ \\
\hline Vogel/Lehner & Vogel/Lehner, Doppelbesteuerungsabkommen, 5. Aufl. 2008 \\
\hline $\mathrm{W} / \mathrm{M}$ & Widmann/Mayer, Umwandlungsrecht (Losebl.) \\
\hline
\end{tabular}

